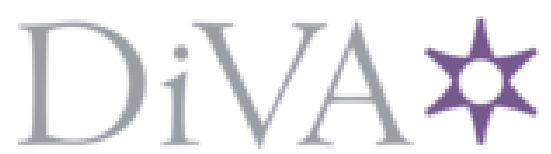

http://www.diva-portal.org

This is the published version of a paper published in International journal of computer integrated manufacturing (Print).

Citation for the original published paper (version of record):

Wang, L., Cai, N., Feng, H-Y. (2009)

Function Blocks Enabled Dynamic Setup Dispatching and Execution Monitoring.

International journal of computer integrated manufacturing (Print), 22(1): 3-12

Access to the published version may require subscription.

N.B. When citing this work, cite the original published paper.

Permanent link to this version:

http://urn.kb.se/resolve?urn=urn:nbn:se:kth:diva-205731 


\title{
Function blocks enabled dynamic set-up dispatching and execution monitoring
}

\author{
Lihui Wang , Ningxu Cai \& Hsi-Yung Feng
}

To cite this article: Lihui Wang , Ningxu Cai \& Hsi-Yung Feng (2009) Function blocks enabled dynamic set-up dispatching and execution monitoring, International Journal of Computer Integrated Manufacturing, 22:1, 3-12, DOI: 10.1080/09511920801911027

To link to this article: http://dx.doi.org/10.1080/09511920801911027

册 Published online: 28 Jan 2009.

Submit your article to this journal $₫$

Џ Article views: 31

Q View related articles $₫$

4 Citing articles: 2 View citing articles 


\title{
Function blocks enabled dynamic set-up dispatching and execution monitoring
}

\author{
Lihui Wang ${ }^{\mathrm{a} *}$, Ningxu Cai ${ }^{\mathrm{a}}$ and Hsi-Yung Feng ${ }^{\mathrm{b}}$ \\ ${ }^{a}$ Department of Mechanical and Materials Engineering, The University of Western Ontario, London, ON N6A 5B9, Canada; \\ ${ }^{b}$ Department of Mechanical Engineering, The University of British Columbia, Vancouver, BC V6T 1Z4, Canada
}

(Received 14 September 2007; final version received 9 January 2008)

\begin{abstract}
With increased product diversification, business globalisation and service outsourcing, manufacturing processes are becoming complicated, especially in job shop operations. Companies must be able to profitably produce in small quantities and make frequent product changeovers to meet customers' needs so as to stay competitive in the global market. The dynamic job shop operations pose new challenges to manufacturing engineers in production planning and control. This paper presents an adaptive approach to dynamic set-up dispatching and execution monitoring by applying function block technology. A two-step decision making of machine-neutral set-up planning and machinespecific set-up merging is proposed in this research. The final set-ups are encapsulated in a set of basic function blocks that can make adaptive decisions at run time using embedded algorithms. The event-driven mechanism of function blocks is further extended for execution monitoring. It is expected that this approach can largely enhance the dynamism and adaptability of job shop operations.
\end{abstract}

Keywords: process planning; function block; set-up dispatching; execution monitoring

\section{Introduction}

From design to manufacturing of a product, a series of tasks must be accomplished, including operation selection, process sequencing, set-up planning, machine/ cutter selection, cutting parameter optimisation, tool path planning, and numerical control (NC) code generation. As commonly referred to as process planning and set-up planning, the tasks are knowledge intensive, complex and dynamic in nature, which make process planning and set-up planning intricate and difficult.

Within the context, process planning is the task that transforms design data into manufacturing processes and determines the optimal sequence of operations. It can also be defined as the act of preparing detailed work instructions to produce a part (Chang et al. 1991), whereas set-up planning is the critical bridge between process planning and operation planning in a machine shop. It is to ensure the stability, and more importantly, to guarantee the precision of the machining process of a part during its machining operations. There are many factors that affect the planning tasks. Part geometry, tolerance, surface finish, raw material, lot size and the available resources (machines, fixtures and cutting tools etc.) all contribute to the decision making during process planning and set-up planning. As set-up planning is closely related to process planning and the two are tightly integrated in our research through function blocks, relevant literature reviews are given below for the ease of understanding of set-up dispatching.

In the past, a number of approaches have been reported in the literature, trying to solve problems in these areas. Earlier efforts include object-oriented approach (Zhang and Lin 1999), Petri-net-based approach (Lee and Jung 1995), artificial-neural-networkbased approach (Devireddy and Ghosh 1999), knowledge-based approach (Stori and Wright 1996), genetic-algorithm-based approach (Zhang et al. 1997), feature-driven approach (Wang and Norrie 2001), and agent-based approach (Wang and Shen 2003). These reported approaches and their combinations have been applied to several specific problem domains, such as setup planning (Ong and Nee 1996), process sequencing (Yeo et al. 1998), tool selection (Lim et al. 2001), cutting parameter selection (Arezoo et al. 2000), and tool path planning (Boogert et al. 1996).

More specifically in the area of set-up planning, there are two major constraints coming from design specifications and manufacturing resources. Most existing set-up planning methods attempted to satisfy the first constraint. Zhang and Lin (1999) introduced the concept of hybrid graph and used tolerance as the critical constraint for set-up planning. Zhang (2001) applied tolerance decomposition, fixture design and manufacturing resource capability to set-up planning. Ong et al. (2002) proposed a hybrid approach to set-up

*Corresponding author. Email: lwang35@uwo.ca 
planning optimisation using genetic algorithms (GAs) and simulated annealing, based on a precedence relationship matrix. An integrated approach to automatic set-up planning was also presented by Huang and $\mathrm{Xu}$ (2003), trying to systematically consider various components: geometry, precedence constraint, kinematics, force and tolerance. More recently, Gologlu (2004) used component geometry, dimensions and tolerances to extract constraint-imposed precedence relations between features, and took fixturing strategies into consideration. The second constraint was normally considered at optimisation stage in terms of cost, quality, lead time, and agility, but under an assumption of given machine tools. In other words, two constraints of set-up planning are treated separately; thus, the search space is narrowed before the search begins (Ong et al. 2002). Recent efforts include set-up grouping strategies for make span minimisation (Yilmaz et al. 2007) and automated set-up planning at both single part level and machine station level (Yao et al. 2007). From the literature, it is evident that the flexibility of set-up planning has not been fully addressed, especially from the viewpoint of integration of set-up planning and set-up dispatching. In other words, set-up planning was done without considering the current availability of machines for set-up dispatching. In order to address the set-up flexibility, machine availability/capability and its dynamic scheduling have to be considered during set-up formation, sequencing, and optimisation.

From the literature, it is also evident that existing research on process planning and set-up planning is mainly focused on analysis of design specifications of workpiece, based on which an optimal or near-optimal plan can be generated. However, these approaches merely consider both the part design and available machine capability at the same time. A process/set-up plan generated in advance by such approaches is static in nature and cannot handle unpredictable events (e.g. missing tools, job delay, schedule change etc.) in a changing shop floor environment. Targeting the problem, in this paper, we present an adaptive approach to set-up planning, set-up merging/dispatching, and set-up execution monitoring, enabled by function blocks with embedded process plan and processing algorithms.

The rest of the paper is organised as follows. Section 2 outlines how process data are encapsulated in function blocks during set-up planning, after a brief introduction to the function block concept. Section 3 presents the principles of set-up merging and set-up dispatching based on available machines and according to dynamic scheduling requirements. The execution monitoring techniques enabled by the function blocks are documented in section 4. Finally, our research findings and contributions are summarised in section 5.

\section{Process data encapsulation in function blocks}

\subsection{Function blocks}

The concept of function blocks is described in the IEC 61499 (2005) specification, as an IEC standard for distributed processes and control systems, particularly for PLC (programmable logic controller) control. It is based on an explicit event-driven model and provides for data flow and finite-state automata-based control. Some early research efforts utilising function blocks include holonic control (Wang et al. 2001), reconfiguration of real-time distributed systems (Brennan et al. 2002), function block oriented engineering support systems (Thramboulidis and Tranoris 2001), web-based engineering and maintenance of distributed control systems (Schwab et al. 2005), and OOONEIDA initiative (2007) where function block can serve as a cornerstone for further development of the automation object concept due to its characteristics of portability and reusability (Vyatkin and Christensen 2005).

The function block concept is relevant to machining in data encapsulation and adaptive process plan execution (Wang et al. 2003). The event-driven model of a function block gives a machine more intelligence and autonomy to make decisions on how to adapt a process plan to match the capacity and dynamics of an actual machine. It enables dynamic resource scheduling, set-up dispatching, and execution monitoring. A basic function block can have multiple outputs and can maintain its internal hidden-state information. This means that a function block can generate different outputs (at different time) even if the same inputs are applied. This is of vital importance for automatic cutting-parameter modifications, after a function block has been dispatched to a specific machine, by changing the internal hidden state of the function block. For example, a function block of pocket_milling can be used for roughing and finishing at the same machine, or at different machines, with different cutting parameters and tool paths by adjusting the internal state of the function block to fine tune the algorithms in use. As shown in Figure 1, each machining feature (hole, step, slot, pocket etc.) in our research is mapped to a basic function block that defines fundamental relationships between events and data. (Details of function block mapping can be found in (Wang et al. 2006b).) The needed processing algorithms are encapsulated in the basic function block and they can only be accessed by the basic function block itself. A group of machining features can form a composite function block (a set-up) by combining basic function blocks 
properly. A set of composite function blocks generated at supervisory planning stage can be merged and dispatched to a chosen machine at run-time for detailed operation planning. The raw event inputs of a function block are used to trigger appropriate machining algorithms encapsulated in the function block, while the data inputs are used for operation planning. The event flow between function blocks determines both the machining sequence and the type of machining operations (roughing or finishing etc.).

\subsection{Process data encapsulation}

As mentioned earlier, a machining feature can be mapped to a machining feature function block
(MF-FB). Taking a 4-side pocket machining feature as an example, Figure 2 gives (a) the graphical definition of the corresponding 4-side pocket MFFB, and (b) its event-driven execution control chart.

Here, EMT is the estimated machining time based on the suggested machining data, which is accumulated and relayed along an MF-FB chain; MT is used to store the actual machining time accumulated during function block execution; MAC_ID passes the ID of a selected machine to the MF-FB for machine-specific operation optimisation including cutter selection, cutting parameter generation and optimal tool path planning; OPER tells the MF-FB the type of machining operation such as roughing, semi-finishing or finishing; FB_EXE is a vector storing the execution

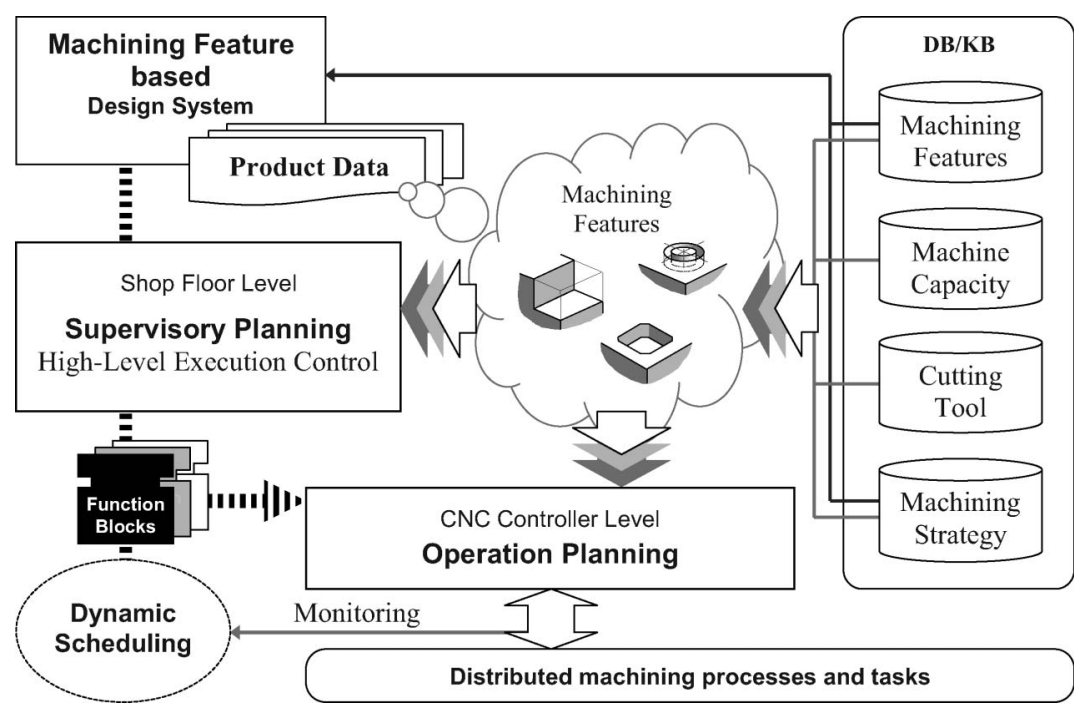

Figure 1. Concept of distributed process planning.

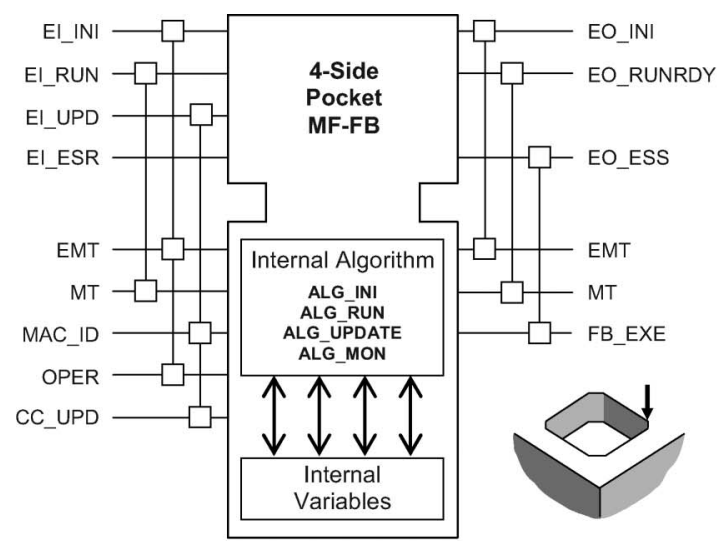

(a) Graphical definition

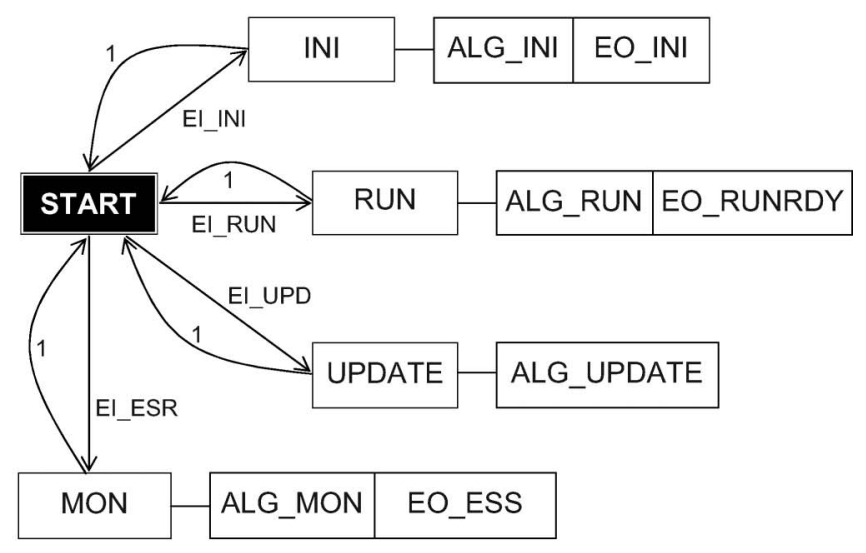

(b) Execution control

Figure 2. A 4-side pocket machining feature function block. 
status and cutting parameters of the function block for monitoring; and CC_UPD is another vector used by a machine operator to override the auto-generated cutting parameters if needed. Based on the external variables and embedded internal variables (not shown in Figure 2(a), e.g. ID of machining feature instance, workpiece material), the four defined algorithms can provide the needed functions upon request.

In Figure 2(b), the START state is an initial idle state ready for receiving event inputs. EI_INI triggers the state transition from START to INI, and when state INI is active, the algorithm ALG_INI is executed for initialisation. Upon completion, A $\bar{L} G_{-}$INI will fire an event EO_INI indicating the success of the initialisation. Similarly, for other state transitions to RUN, UPDATE and MON, different algorithms ALG_RUN (MF-FB execution), ALG_UPDATE (cutting-parameter update), and ALG_MON (MFFB monitoring) are triggered, correspondingly. Note that ALG_MON is triggered to run in a different thread from other algorithms so that a concurrent execution of this algorithm becomes possible. This is important for execution status monitoring of function blocks once dispatched to a machine controller. The cycle time of ALG_MON is customisable, with a default value of $50 \mathrm{~ms}$.

In the execution control chart, an event ' 1 ' means a state transition is always true. That is to say, the state will transit back to the START state and be ready for receiving the next event input. If a START state is not ready, any arrival events (except EI_ESR) will be ignored.

Other required machining information embedded in an MF-FB, such as cutter types for different operations and suggested tool-path patterns are listed in Table 1. With the required machining data and the embedded algorithms, an MF-FB like this is empowered to make adaptive decisions, at run-time, with respect to the assigned resources (e.g. machine and cutters), including calculating optimal cutting conditions, generating tool path according to the suggested tool-path patterns, and estimating or accumulating machining time. In addition to their adaptability and portability, function blocks can also be reused in different machining applications by re-arranging the sequence of predefined function blocks.

\subsection{Function blocks in set-up merging and execution monitoring}

In our research, function blocks are adopted to encapsulate process plans and to enable adaptive setup merging and monitoring during process plan execution.

As shown in Figure 3, function blocks are first generated at the process planning stage, representing 3 -axis based and machine-neutral set-ups. Each basic function block $(\mathrm{FB})$ is mapped to a machining feature (MF) in a particular set-up. During dynamic scheduling when the currently available machines are given, the 3-axis based set-ups (or function block network) are merged to form a smaller number of set-ups for the available machines. The newly merged set-ups must meet the given scheduling requirements on cost, make span and/or machine utilisation before being dispatched to the assigned machines. This is guaranteed by our GA-based algorithms for cross-machine adaptive set-up planning, which has been integrated loosely with a scheduling system and reported separately (Cai et al. 2008). During process plan (or function block) execution, the real-time machining status (including machining feature ID, cutter location, cutting condition and remaining machining time) can be monitored by the event-driven mechanism and the built-in algorithm ALG_MON (see Figure 2) of the function blocks. The monitoring information is crucial to execution control and dynamic scheduling.

Table 1. MF-FB embedded machining information.

\begin{tabular}{|c|c|c|c|}
\hline & Operation & Cutter type \\
\hline Feature type & $\begin{array}{l}f: \text { Feed per tooth } \\
n: \text { Flute number } \\
L: \text { Tool-path length }\end{array}$ & $\begin{array}{l}\text { Roughing } \\
\text { Finishing }\end{array}$ & $\begin{array}{l}\text { Square end mill } \\
\text { Square end mill (diameter smaller } \\
\text { than twice the corner radius) }\end{array}$ \\
\hline
\end{tabular}

Suggested tool-path patterns

(1)

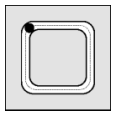

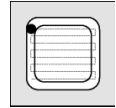

(2)
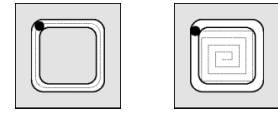

Machining time estimation: $T=L /(f \cdot n \cdot r p m)$ 


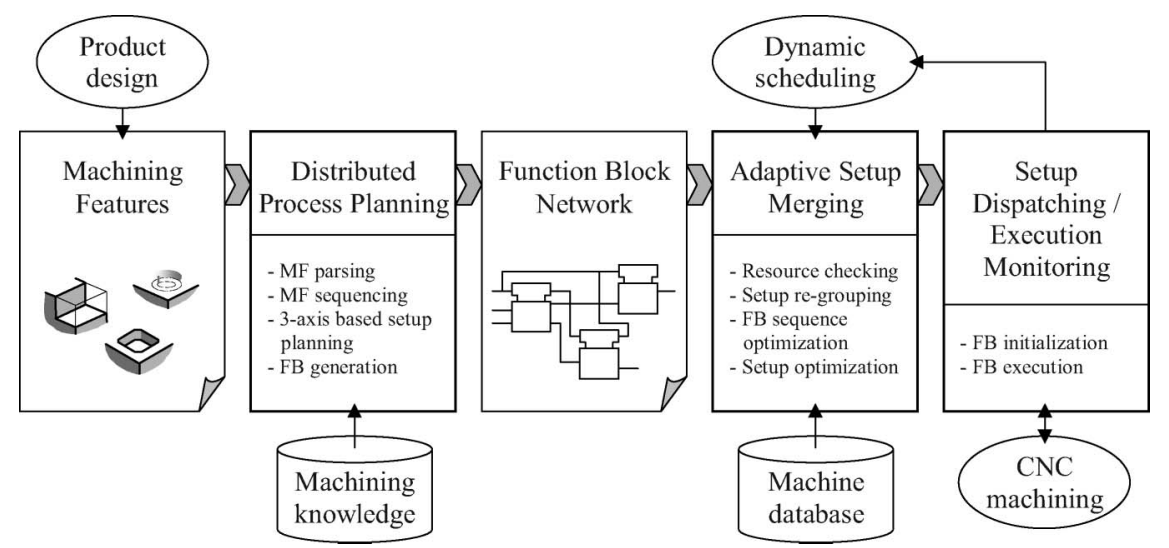

Figure 3. Function blocks in setup merging and execution monitoring.

\section{Adaptive set-up merging and dispatching}

In our research, the supervisory planning as shown in Figure 1 can generate a generic sequence plan. At the same time, a set-up plan is created for 3-axis machines, as their configurations form the basis of other machines having more axes. However, set-up merging is required for a 4- or 5-axis machine to best utilise the capability of the higher-end machine, after the non 3axis machine is selected. This is explained through an example.

According to our geometric reasoning rules (Wang et al. 2006a), a 3-axis based generic set-up plan of a test part (shown in Figure 4(a)) with 26 machining features can be generated. It consists of 5 set-ups, each of which contains a set of partially-sequenced machining features, as shown in Figure 4(b). The light grey areas are set-ups and the dark grey areas indicate the feature groups sharing the same cutting tools. Each 3-axis based set-up can be represented by a unique unit vector $\boldsymbol{u}$ indicating its tool-access direction (TAD). When a 5axis machine $\{X, Y, Z, A$ (around $X), B$ (around $Y$ ) $\}$ is selected, more than one of the 3 -axis based set-ups of the test part may have a chance to be machined in one final set-up through set-up merging.

The set-up merging examines whether other setups can be included in a final set-up by checking the unit vector $\boldsymbol{u}$ of each set-up against the tool-orientation space (TOS) of the selected machine. The procedure is straightforward by following two steps and their iterations, i.e. (1) aligning the locating direction of a final set-up to the spindle axis $Z$, and (2) searching for an orientation that includes a maximum number of 3 -axis based set-ups by rotating the part around the spindle axis $Z$. This merging process is repeated for all set-ups until a minimum number of 5-axis based final set-ups can be reached. Since the first step can be done easily by using matrix transformation, we only provide details on the second step due to page limitation.

Figure 5(a) shows a typical scenario, where a set-up has been aligned with $-Z$ axis and another 3 -axis based set-up with a tool-access direction $\boldsymbol{u}_{\boldsymbol{i}}\left(x_{i}, y_{i}, z_{i}\right)$ is under consideration. The goal is to rotate the vector $\boldsymbol{u}_{\boldsymbol{i}}$ (or the test part) around $Z$ and at the same time determine a mergable range (or ranges) within $2 \pi$, where $\boldsymbol{u}_{\boldsymbol{i}}$ can fit in the TOS of the machine. The TOS is represented as a spherical surface patch denoted by EFGH in Figure 5(a).

As shown in Figure 5(a), the spherical coordinates of $\boldsymbol{u}_{\boldsymbol{i}}$ are $\left(1, \gamma_{i}, \theta_{i}\right)$. By rotating $\boldsymbol{u}_{\boldsymbol{i}}$ around $Z$, a circle $\boldsymbol{C}_{\boldsymbol{i}}$ is obtained.

$$
\left\{\begin{array}{l}
x_{i}=\sin \theta_{i} \cos \gamma_{i} \\
y_{i}=\sin \theta_{i} \sin \gamma_{i} \\
z_{i}=-\cos \theta_{i}
\end{array}\right.
$$

where, $\theta_{i}$ is a constant and $\gamma_{i} \in[0,2 \pi]$. The $\boldsymbol{C}_{\boldsymbol{i}}$ may intersect with the spherical surface patch EFGH defined by

$$
\begin{aligned}
& \text { EF: } \phi_{A}=\Phi_{A}^{+}, \phi_{B} \in\left[\Phi_{B}^{-}, \Phi_{B}^{+}\right] \\
& \text {FG: } \phi_{B}=\Phi_{B}^{+}, \phi_{A} \in\left[\Phi_{A}^{-}, \Phi_{A}^{+}\right] \\
& \text {GH: } \phi_{A}=\Phi_{A}^{-}, \phi_{B} \in\left[\Phi_{B}^{-}, \Phi_{B}^{+}\right] \\
& \text {HE: } \phi_{B}=\Phi_{B}^{-}, \phi_{A} \in\left[\Phi_{A}^{-}, \Phi_{A}^{+}\right]
\end{aligned}
$$

where, $\left[\Phi_{A}^{-}, \Phi_{A}^{+}\right]$and $\left[\Phi_{B}^{-}, \Phi_{B}^{+}\right]$are the motion ranges of axes $A$ and $B$, respectively. For $\phi_{A}=\Phi_{A}^{+}$and $\phi_{B} \in\left[\Phi_{B}^{-}, \Phi_{B}^{+}\right]$,

$$
|z|=\sqrt{\frac{\left(\cos \left(\Phi_{A}^{+}\right)\right)^{2}}{1+\left(\cos \left(\Phi_{A}^{+}\right) * \tan \left(\phi_{B}\right)\right)^{2}}}, \phi_{B} \in\left[\Phi_{B}^{-}, \Phi_{B}^{+}\right]
$$


If $\left|z_{i}\right|<|z|_{\min }$, the segment $\mathbf{E F}:\left\{\phi_{A}=\Phi_{A}^{+}, \phi_{B} \in\right.$ $\left.\left[\Phi_{B}^{-}, \Phi_{B}^{+}\right]\right\}$and the circle $C_{\boldsymbol{i}}$ has no intersection. If $z_{i}<0$ and $\left|z_{i}\right|>|z|_{\max }$, the segment $\mathbf{E F}$ and circle $\boldsymbol{C}_{\boldsymbol{i}}$ intersect over the entire range of $[0,2 \pi]$. Otherwise, if $z_{i}<0$ and $|z|_{\min }<\left|z_{i}\right|<|z|_{\max }, \mathbf{E F}$ and $\boldsymbol{C}_{\boldsymbol{i}}$ intersect with each other along the edge of the TOS. Figure 5(b) gives the mergable range of the case shown in

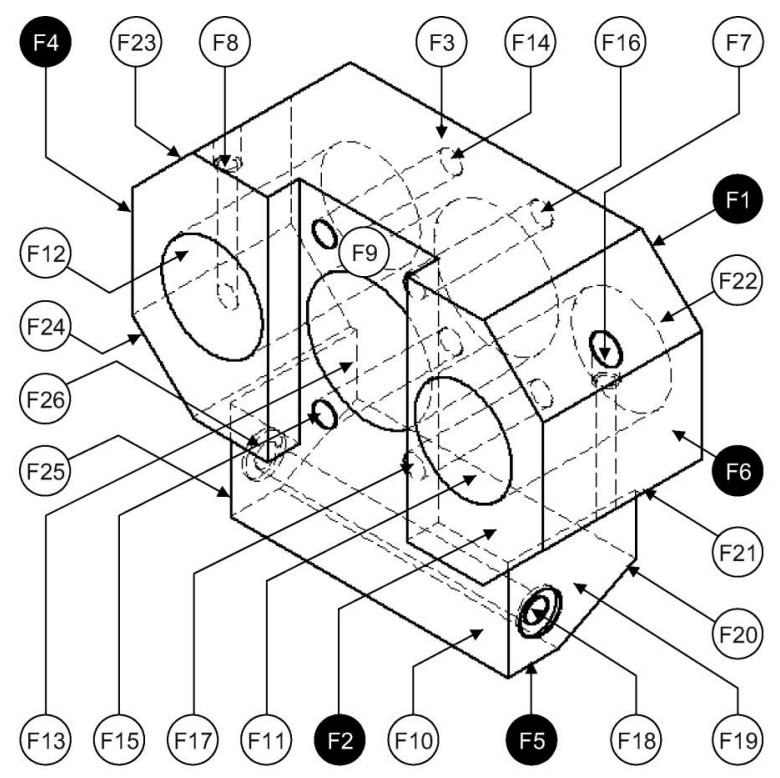

(a) A test part

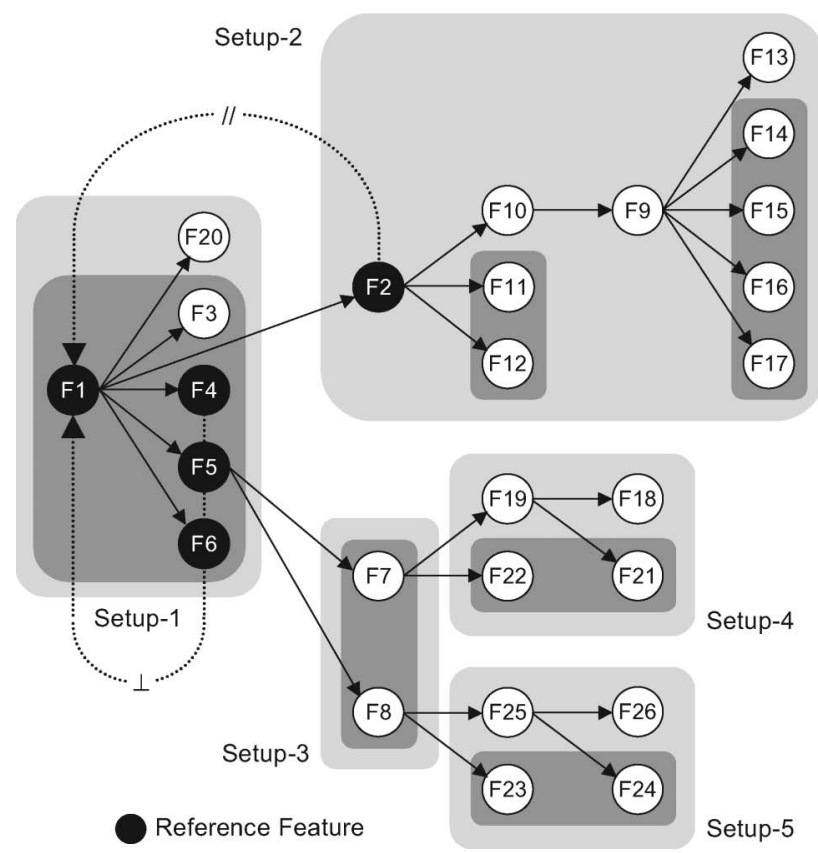

(b) 3-axis based setups

Figure 4. A test part with 5 set-ups after applying geometric reasoning rules.
Figure 5(a), which can be calculated for every 3-axis based set-up. As shown in Figure 6, a pose (position and orientation) of the test part that provides the most overlapping mergable range determines a 5 -axis based set-up.

Figure 7 depicts the result of the test part after the five generic set-ups in Figure 4(b) have been merged to

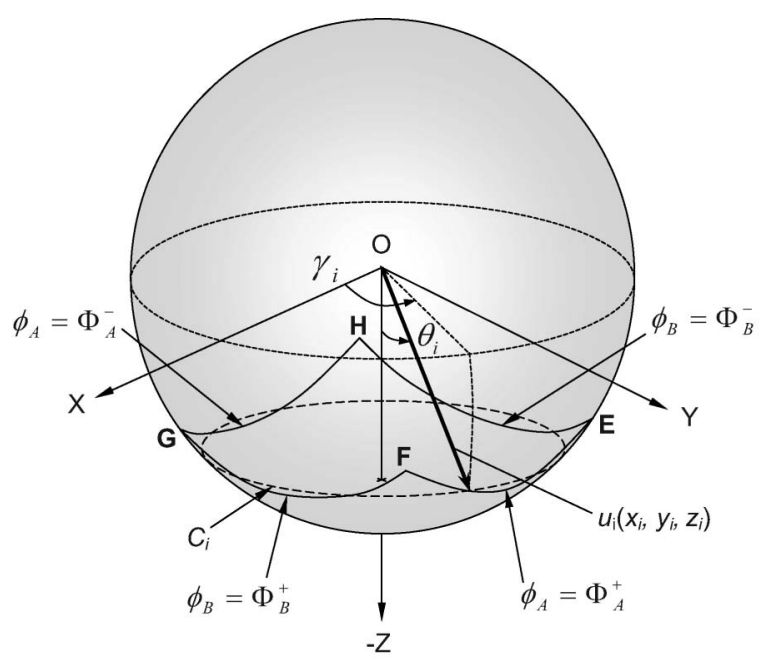

(a) Searching for setup mergability in TOS

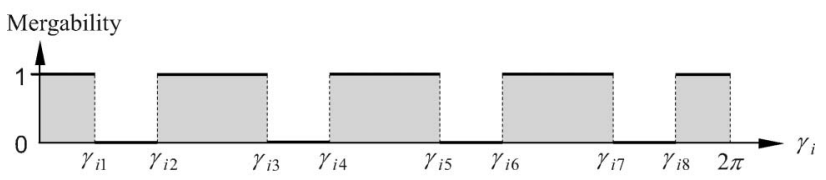

(b) Mergable range of a setup with TAD $\boldsymbol{u}_{i}$

Figure 5. Set-up merging for a 5-axis machine.

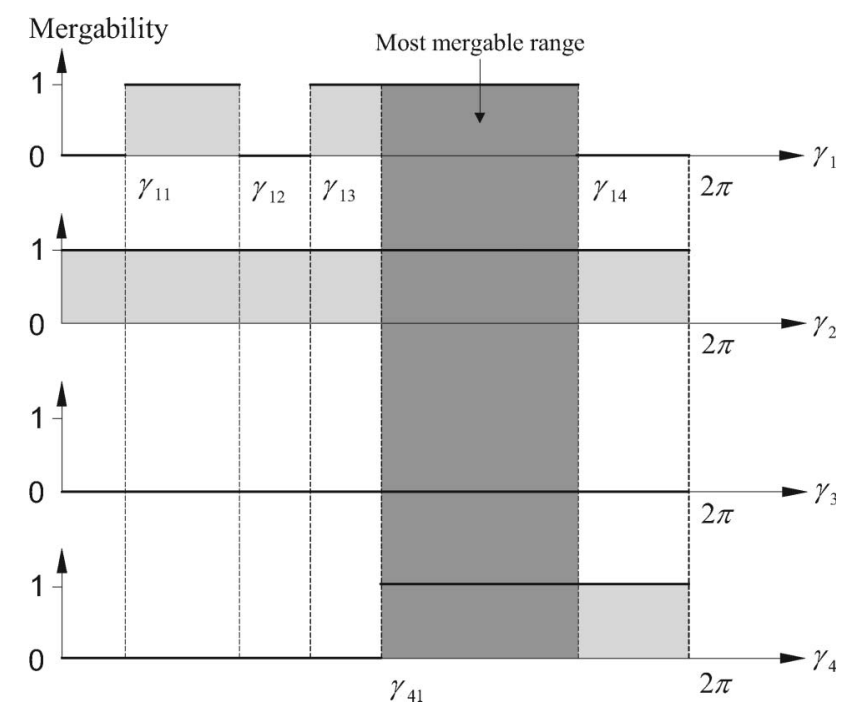

Figure 6. Determination of a most mergable range. 
two set-ups (light grey areas) for the 5-axis machine. This final set-up plan in the form of two composite function blocks is then dispatched to the chosen machine.

\section{Function block execution monitoring}

After a merged set-up (e.g. Set-up-2 in Figure 7) has been dispatched to its dedicated machine, detailed

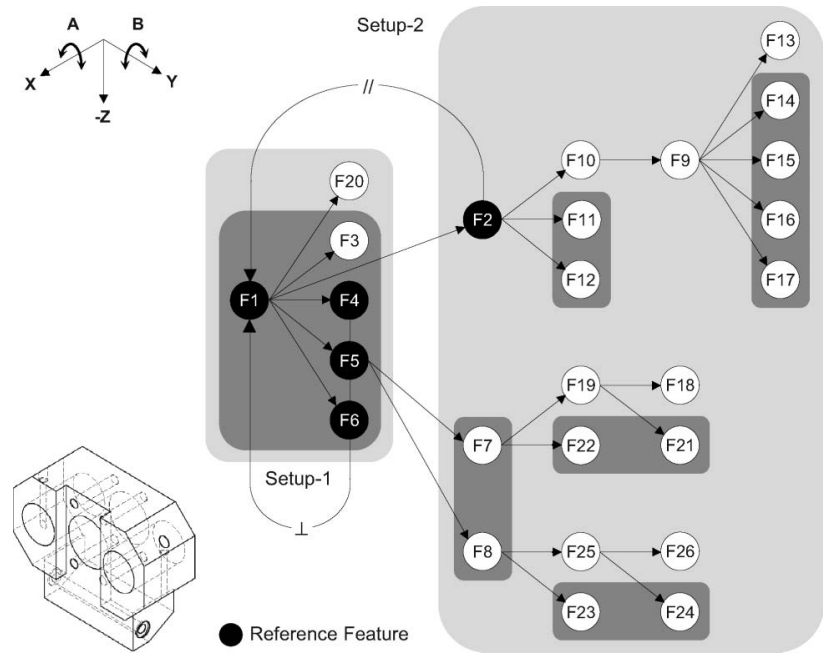

Figure 7. Results of set-up merging for a 5-axis machine. operation planning is performed. The algorithm ALG_INI in each MF-FB can choose a cutter, determine a set of cutting parameters, plan tool paths, and generate optional G-code for conventional machines. A knowledge base that contains suggested cutting tools and tool-path patterns for each $\mathrm{MF}-\mathrm{FB}$ is used to facilitate operation planning. Although the run-time initialisation runs transparently in a controller, a user interface is implemented to visualise the process and to verify the concept, as shown in Figure 8. The machining sequence $\mathbf{1}$ and set-up 2 are derived based on datum references, manufacturing constraints, and the geometric reasoning rules, while other machining data 35 are derived by individual MF-FBs. The data in $\mathbf{3}$ and $\mathbf{4}$ is used to cut corresponding machining features. The G-code $\mathbf{5}$ of a set-up (set-up-5 merged into base set-up-2 in this case) is generated for the selected machine, by assembling blocks of G-code of each machining feature in the order of the defined sequence. Note that the function of G-code generation in function blocks is to best utilise legacy machines that do not support function blocks. The optional G-code generation is triggered when the set-up is dispatched to a non OAC (open architecture controller) based machine (or a legacy machine that only accepts G-code).

Unlike conventional process planning and setup planning systems, our function block enabled

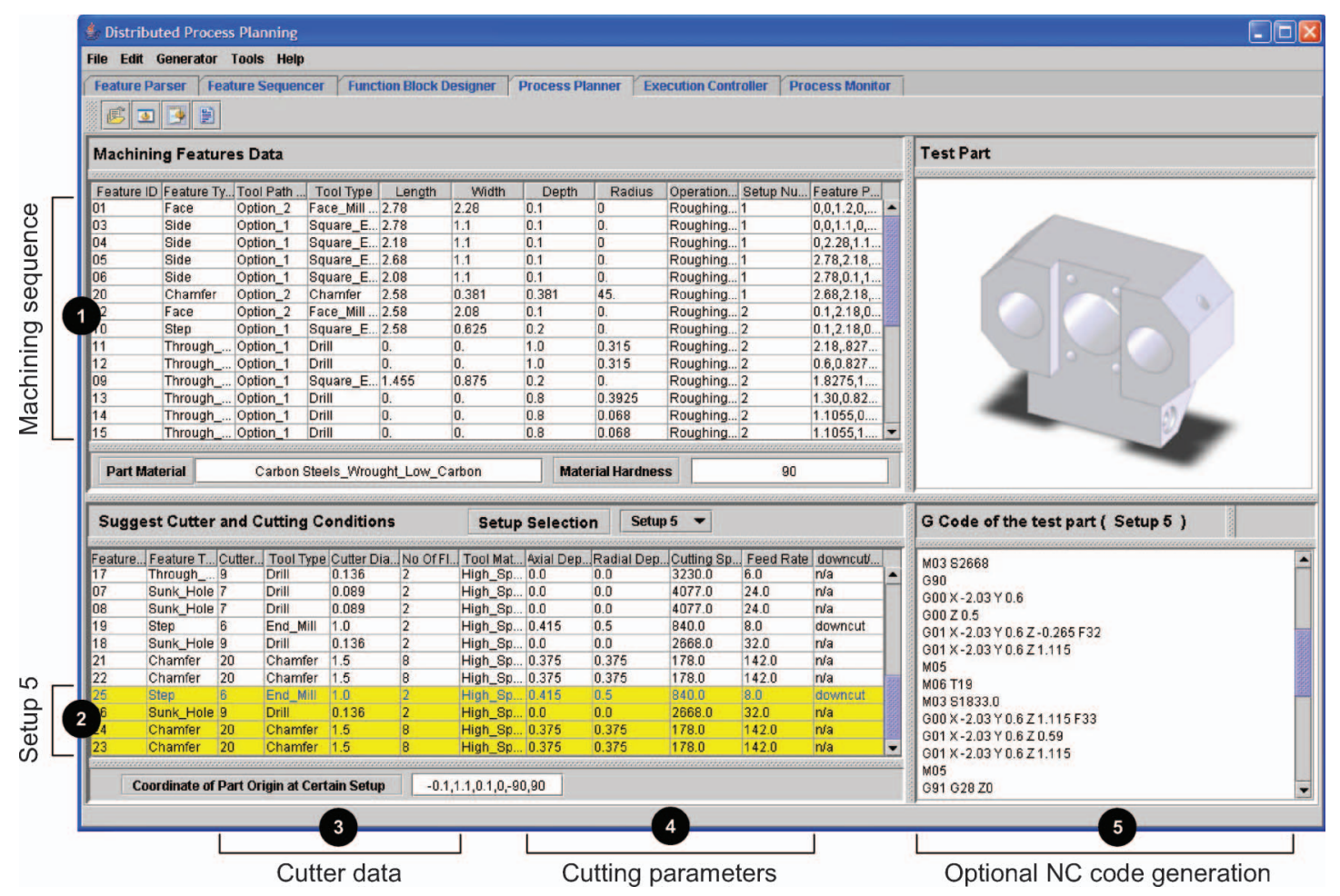

Figure 8. Detailed machining data derived by function block embedded algorithms. 
approach can provide bi-directional information flow. The monitoring information from bottom up adds value to adaptive set-up planning and is of vital importance for shop-floor execution control and realtime scheduling. Our current implementation enables both remote monitoring through an execution control

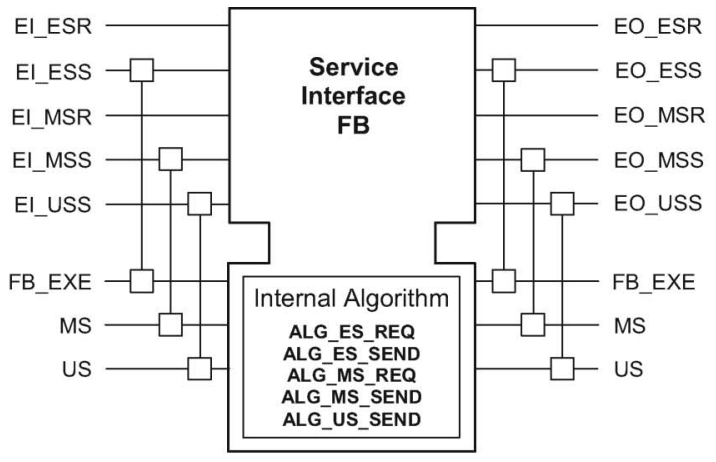

(a) Service interface function block

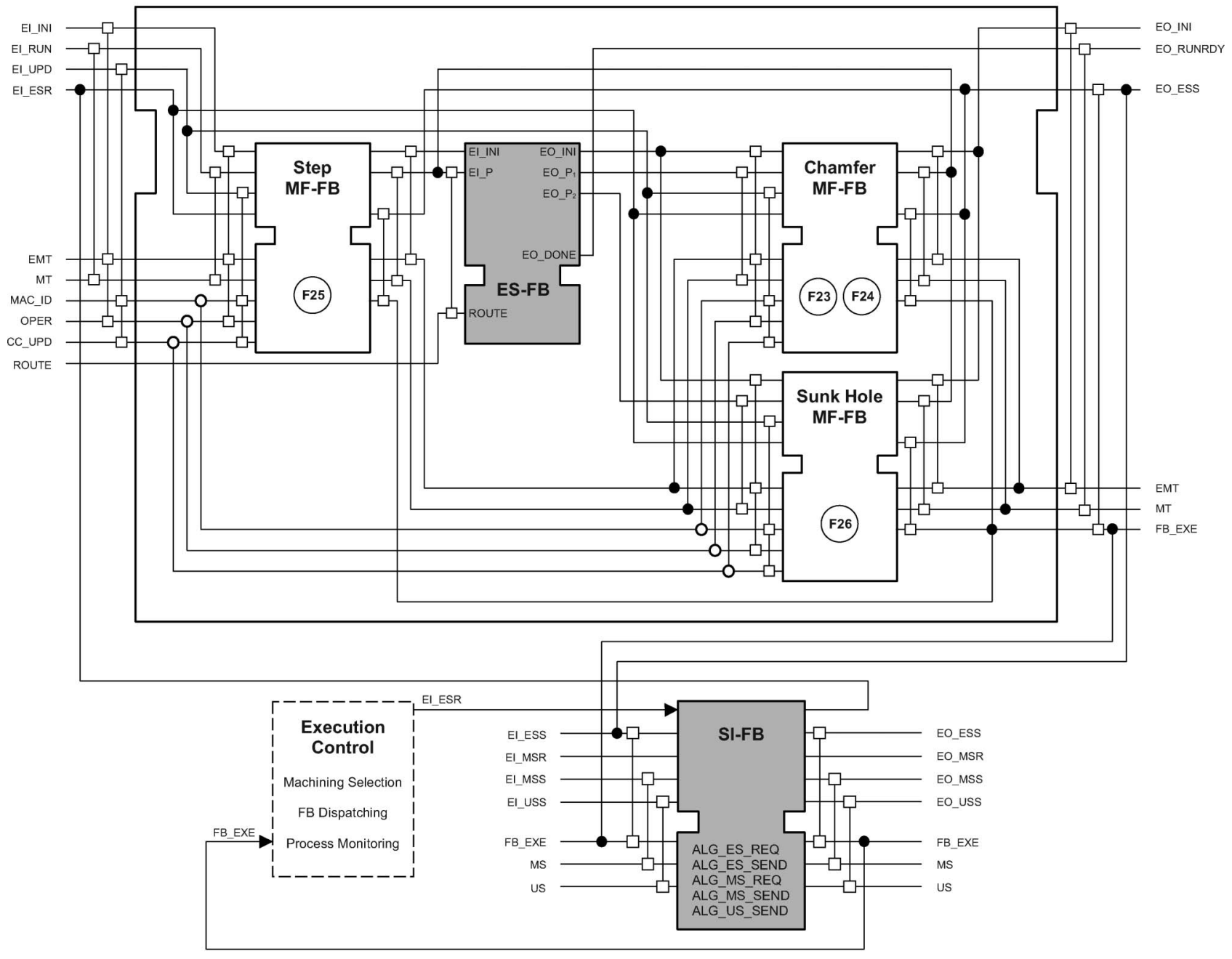

(b) SI-FB plugged to MF-FBs for execution monitoring

Figure 9. Execution monitoring by a service interface function block. 

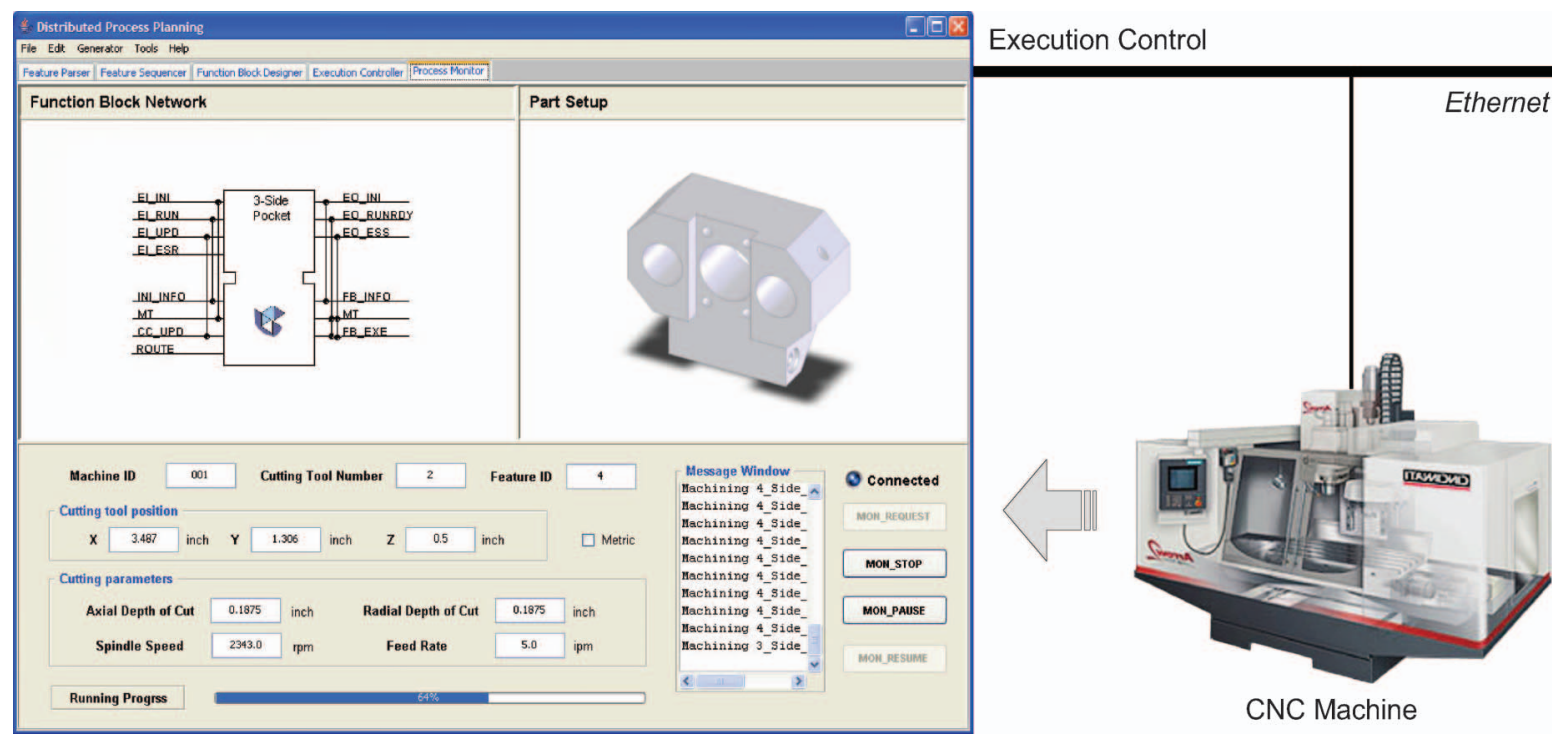

Figure 10. Real-time function block execution monitoring.

module and local monitoring beside a machine using the operation planning module. TCP/IP through sockets is used for data communication. For execution monitoring, a service interface function block (SI-FB) is designed as shown in Figure 9(a) with five needed algorithms embedded. They are responsible for requesting and reporting execution status (ES), machining status (MS), and unexpected situation (US) from MF-FBs and to the high-level execution control module (see Figure 1), respectively. The SI-FB can be chained with other MF-FBs, as shown in Figure 9(b), while interfacing and sharing run-time process data with the dedicated machine and the execution control module. Per request of the execution control module, the SI-FB will pass the request (EI_ESR, execution status request) to the composite function block in Figure 9(b), which will then return an array of FB_EXE containing run-time execution status back to the SI-FB and finally to the execution control module. Figure 10 demonstrates one scenario for remote monitoring of the test part. Once a request is sent to the composite function block that runs on a specific machine, its run-time execution status including current machining feature ID, cutter location, cutting conditions and job completion rate (\%) will be sent back to the requester, continuously at a preset but customisable time interval.

\section{Conclusions}

This paper presents a function block enabled approach to meet the challenges of process planning and set-up planning for dynamic job shop operations. The novelty of this research is the design and integration of event-driven function blocks with process/set-up planning and execution monitoring. Rather than the static how to do defined in the G-code by ISO-6983, our function blocks only tell a machine what to do, leaving the machine-specific information of how to do to the function block embedded algorithms. Using such embedded algorithms, the function blocks are able to generate and/or modify the machine-specific data at run time to better adapt to any shop floor uncertainty. In this research, a process plan of a given part is divided into set-ups, each of which is a composite function block. Furthermore, in order to achieve generality of a set-up plan, 3-axis machines are chosen for generic set-up planning that only needs to be done once and in advance. Set-up merging is performed when a non 3-axis machine is selected before dispatching. The two-step decision making makes this approach unique and efficient for run-time processing. Powered by the adaptive planning and real-time monitoring, it is expected that this novel approach can largely increase the flexibility and adaptability of job shop operations, especially in fluctuating environments.

Our future plan of this research includes controllerside implementations that eventually make the defined function blocks a part of the controller's firmware for real shop floor applications.

\section{References}

Arezoo, B., Ridgway, K., and Al-Ahmari, A.M.A., 2000. Selection of cutting tools and conditions of machining operations using an expert system. Computers in Industry, $42,43-58$. 
Boogert, R.M., Kals, H.J., and van Houten, F.J., 1996. Tool paths and cutting technology in computer-aided process planning. International Journal of Advanced Manufacturing Technology, 11, 186-197.

Brennan, R.W., et al., 2002. A reconfigurable concurrent function block model and its implementation in real-time Java. Integrated Computer-Aided Engineering, 9, 263279.

Cai, N., Wang, L., and Feng, H.-Y., 2008. GA based adaptive set-up planning toward process planning and scheduling integration. International Journal of Production Research, Published online 22 January 2008. doi 10.1080/00207540701663516.

Chang, T.-C., Wysk, R.A., and Wang, H.-P., 1991. Computer-aided manufacturing. New Jersey: Prentice Hall.

Devireddy, C.R. and Ghosh, K., 1999. Feature-based modelling and neural network-based CAPP for integrated manufacturing. International Journal of Computer Integrated Manufacturing, 12, 61-74.

Gologlu, C., 2004. Machine capability and fixturing constraints-imposed automatic machining setups generation. Journal of Materials Processing Technology, 148, 83-92.

Huang, S.H. and Xu, N., 2003. Automatic set-up planning for metal cutting: an integrated methodology. International Journal of Production Research, 41, 4339-4356.

International Electrotechnical Commission, 2005. IEC 61499, International Standard of Function Blocks - Part 1: Architecture, Geneva, Switzerland: IEC, pp. 1-111.

Lee, K.Y. and Jung, M.Y., 1995. Flexible process sequencing using Petri net theory. Computers and Industrial Engineering, 28, 279-290.

Lim, T., et al., 2001. Optimising tool selection. International Journal of Production Research, 39, 1239-1256.

OOONEIDA, 2007 [online]. Available from: http://www. oooneida.info/.

Ong, S.K., Ding, J., and Nee, A.Y.C., 2002. Hybrid GA and SA dynamic set-up planning optimisation. International Journal of Production Research, 40, 4697-4719.

Ong, S.K. and Nee, A.Y.C., 1996. Fuzzy-set-based approach for concurrent constraint setup planning. Journal of Intelligent Manufacturing, 7, 107-120.

Schwab, C., Tangermann, M., and Ferrarini, L., 2005. Web based methodology for engineering and maintenance of distributed control systems: the TORERO approach. In: Proceedings of the 3rd IEEE International Conference on Industrial Informatics, 2005, Perth, Australia.

Stori, J.A. and Wright, P.K., 1996. A knowledge-based system for machining operation planning in feature based, open-architecture manufacturing. In: Proceedings of ASME DETC'96. Irvine, CA.

Thramboulidis, K. and Tranoris, C., 2001. An architecture for the development of function block oriented engineering support systems. In: Proceedings of IEEE International Conference on Computational Intelligence in Robotics and Automation, Banff, Canada.
Vyatkin, V.V. and Christensen, J.H., 2005. OOONEIDA: An open, object-oriented knowledge economy for intelligent industrial automation. IEEE Transaction on Industrial Informatics, 1, 4-17.

Wang, L., et al., 2001. Realising holonic control with function blocks. Integrated Computer-Aided Engineering, 8, 81-93.

Wang, L., et al., 2006a. Enriched machining feature based reasoning for generic machining process sequencing. International Journal of Production Research, 44, 14791501 .

Wang, L., Feng, H.-Y., and Cai, N., 2003. Architecture design for distributed process planning. Journal of Manufacturing Systems, 22, 99-115.

Wang, L., Jin, W., and Feng, H.-Y., 2006b. Embedding machining features in function blocks for distributed process planning. International Journal of Computer Integrated Manufacturing, 19, 443-452.

Wang, L. and Norrie, D.H., 2001. Process planning and control in a holonic manufacturing environment. Journal of Applied Systems Studies, 2, 106-126.

Wang, L. and Shen, W., 2003. DPP: an agent-based approach for distributed process planning. Journal of Intelligent Manufacturing, 14, 429-440.

Yeo, S.H., Ngoi, B.K.A., and Chen, H., 1998. Process sequence optimisation based on a new cost-tolerance model. Journal of Intelligent Manufacturing, 9, 29-37.

Yao, S., et al., 2007. Computer aided manufacturing planning for mass customisation: part 2, automated setup planning. International Journal of Advanced Manufacturing Technology, 32, 205-217.

Yilmaz, I.O., et al., 2007. Development of group setup strategies for makespan minimisation in PCB assembly. International Journal of Production Research, 45 (4), 871897.

Zhang, Y., et al., 1999. Object oriented manufacturing resource modelling for adaptive process planning. International Journal of Production Research, 37, 4179-4195.

Zhang, H.-C. and Lin, E., 1999. A hybrid-graph approach for automated setup planning in CAPP. Robotics and Computer-Integrated Manufacturing, 15, 89-100.

Zhang, Y., 2001. Graph-based set-up planning and tolerance decomposition for computer-aided fixture design. International Journal of Production Research, 39, 3109-3126.

Zhang, F., Zhang, Y.F., and Nee, A.Y.C., 1997. Using genetic algorithms in process planning for job shop machining. IEEE Transactions on Evolutionary Computation, 1, 278-289. 